\title{
Fabrication methods for a quantum cascade photonic crystal surface emitting laser
}

\section{Citation}

Tennant, D. M., R. Colombelli, K. Srinivasan, M. Troccoli, O. Painter, C. Gmachl, F. Capasso, A. M. Sergent, D. L. Sivco, and A. Y. Cho. 2003. "Fabrication Methods for a Quantum Cascade Photonic Crystal Surface Emitting Laser." Journal of Vacuum Science \& Technology B: Microelectronics and Nanometer Structures 21 (6): 2907. https://doi.org/10.1116/1.1627815.

\section{Permanent link}

http://nrs.harvard.edu/urn-3:HUL.InstRepos:41372485

\section{Terms of Use}

This article was downloaded from Harvard University's DASH repository, and is made available under the terms and conditions applicable to Other Posted Material, as set forth at http:// nrs.harvard.edu/urn-3:HUL.InstRepos:dash.current.terms-of-use\#LAA

\section{Share Your Story}

The Harvard community has made this article openly available. Please share how this access benefits you. Submit a story.

Accessibility 


\title{
Fabrication methods for a quantum cascade photonic crystal surface emitting laser
}

\author{
D. M. Tennant \\ Bell Laboratories, Lucent Technologies, Murray Hill, New Jersey 07974 and New Jersey Nanotechnology \\ Consortium, Murray Hill, New Jersey 07974 \\ R. Colombelli \\ Bell Laboratories, Lucent Technologies, Murray Hill, New Jersey 07974 \\ K. Srinivasan \\ Department of Applied Physics, California Institute of Technology, Pasadena, California 91125 \\ M. Troccoli \\ Division of Engineering and Applied Sciences, Harvard University, Cambridge, Massachusetts 02138 \\ O. Painter \\ Department of Applied Physics, California Institute of Technology, Pasadena, California 91125 \\ C. Gmachi \\ Bell Laboratories, Lucent Technologies, Murray Hill, New Jersey 07974 \\ F. Capasso \\ Division of Engineering and Applied Sciences, Harvard University, Cambridge, Massachusetts 02138
}

A. M. Sergent

Bell Laboratories, Lucent Technologies, Murray Hill, New Jersey 07974

and New Jersey Nanotechnology Consortium, Murray Hill, New Jersey 07974

D. L. Sivco and A. Y. Cho

Bell Laboratories, Lucent Technologies, Murray Hill, New Jersey 07974

(Received 26 June 2003; accepted 29 September 2003; published 9 December 2003)

\begin{abstract}
Conventional quantum cascade (QC) lasers are intrinsically edge-emitting devices with mode confinement achieved via a standard mesa stripe configuration. Surface emission in edge emitting QC lasers has therefore necessitated redirecting the waveguided laser emission using a second order grating. This paper describes the methods used to fabricate a 2D photonic crystal (PC) structure with or without a central defect superimposed on an electrically pumped QC laser structure with the goal of achieving direct surface emission. A successful systematic study of PC hole radius and spacing was performed using e-beam lithography. This PC method offers the promise of a number of interesting applications, including miniaturization and integration of QC lasers. (C) 2003 American Vacuum Society. [DOI: 10.1116/1.1627815]
\end{abstract}

\section{INTRODUCTION}

Since their invention in 1994 (Ref. 1) quantum cascade (QC) lasers have rapidly established themselves as tunable coherent sources in the mid-infrared (mid-IR) range of the electromagnetic spectrum. ${ }^{2}$ Improvements since their first introduction include room temperature operation, $\mathrm{THz}$ emission, and fabrication in a wide variety of binary and ternary III-V heteroepitaxial semiconductor material systems. Applications range from trace gas detection, industrial process monitoring and control, to molecular spectroscopy.

QC lasers are unique among lasers since they use an intraband rather than an interband optical transition. Electrons do not recombine with valence band holes: they instead undergo a transition between the subbands of a multiquantum well structure. The emission wavelength is thus not determined by the material, but by the layer thicknesses in the heterostructure. ${ }^{2}$ For this reason QC lasers with varying layer composition can cover a very broad wavelength range [3.5 $\mu \mathrm{m}$ to $24 \mu \mathrm{m}$ in the InGaAs/AlInAs system; ${ }^{3}$ up to $87 \mu \mathrm{m}$ in GaAs/AlGaAs (Ref. 4)]. Since intersubband transitions are transverse magnetic (TM) polarized (i.e., the electric field is orthogonal to the layers), conventional QC lasers are intrinsically edge-emitting devices with mode confinement achieved via a standard mesa stripe configuration. Surface emission in edge emitting QC lasers has therefore necessitated redirecting the waveguided laser emission using a second order grating superimposed on standard edge-emitting QC lasers. ${ }^{5}$ The second order distributed feedback (DFB) partially redirects the beam in the vertical direction. There are, however, disadvantages with this approach. Due to $k$-vector conservation there is emission into the substrate, the redirection efficiency is quite low, and the devices that employ this technique typically exhibit broad far-field emission patterns in the direction orthogonal to the waveguide. More generally, the technique does not allow flexibility in engineering the far-field. Finally, the relatively large dimensions of the devices (due to the presence of the necessary FabryPerot cavity) preclude any application that requires miniaturization.

We propose a different approach that allows us to manu- 
facture electrically pumped QC lasers as conventional surface-emitting lasers. Our approach consists of fabricating the QC laser cavity as a (hexagonal) 2D photonic crystal (PC). Photonic crystals can create a range of forbidden frequencies (bandgap) and can dramatically alter the photonic density of states. ${ }^{6,7}$ Planar PC devices employ a 2D photonic lattice with a conventional slab vertical waveguide. They are now considered a particularly appealing device architecture due to the maturity of planar fabrication methods. ${ }^{8-10}$ Twodimensional PC slab waveguide lasers can be divided in two families: defect mode lasers and band-edge mode lasers. Defect mode lasers operate at frequencies inside the photonic band gap, and the optical mode is strongly localized on a defect intentionally incorporated in the lattice. ${ }^{11}$ Band-edge mode lasers, on the other hand, operate in regions of energymomentum space that have a very small group velocity, as a result of the 2D distributed feedback of the photonic lattice. ${ }^{12}$ To date, defect mode lasers with cavity dimensions at the wavelength scale have been demonstrated in an opticallypumped configuration only. ${ }^{11,13}$ In contrast, band-edge lasers typically have been modeled and fabricated as broad-area devices involving several hundred PC periods, and they are pumped either optically or electrically. ${ }^{12,14,15}$

In this work, we discuss the processes used to fabricate an electrically pumped electroluminescent structure which comprises a unipolar intraband active region and a microcavity resonator. The resonator includes a $2 \mathrm{D}$ hexagonal array PC that exhibits resonant modes. The etch depth in the PC area extends through the active region and thereby operates in the strong grating regime. The dimensions are designed so that the array acts as a micro-cavity resonator in which the resonant modes fall within the gain spectrum and such that light propagation includes a significant component that is directed transverse to the axis of the resonator, thus functioning as a surface-emitting intersubband laser. Emission is then vertical from a compact space allowing arrays of devices to be produced on a single chip. We also expect it will be possible to improve the far-field emission patterns and even to specifically engineer the emission patterns by tailoring the PC design, and to reduce the emission into the substrate. ${ }^{16}$

\section{FABRICATION}

The QC heterostructure material used is based on a 3-well vertical design of the active region. ${ }^{17}$ It is grown by molecular beam epitaxy (MBE) on a semi-insulating InP substrate. The epitaxial material consists of an InGaAs/AlInAs layer $2.5 \mu \mathrm{m}$ thick capped by an $n^{++}$-InGaAs layer for contact purposes. No AlInAs claddings are grown on top of the active region, since a surface plasmon waveguide is employed. ${ }^{18}$ The nominal emission wavelength is $7.8 \mu \mathrm{m}$.

The major processing steps are shown in Fig. 1 and are briefly described here. A hard mask layer of silicon dioxide $250-750 \mathrm{~nm}$ is deposited by plasma-enhanced chemical vapor-deposition (PECVD). The PC pattern is written using electron beam lithography on a JEOL 9300FS $100 \mathrm{kV}$ system. We used a commercially available environmentally stabilized chemically amplified photoresist (ESCAP), UV 113
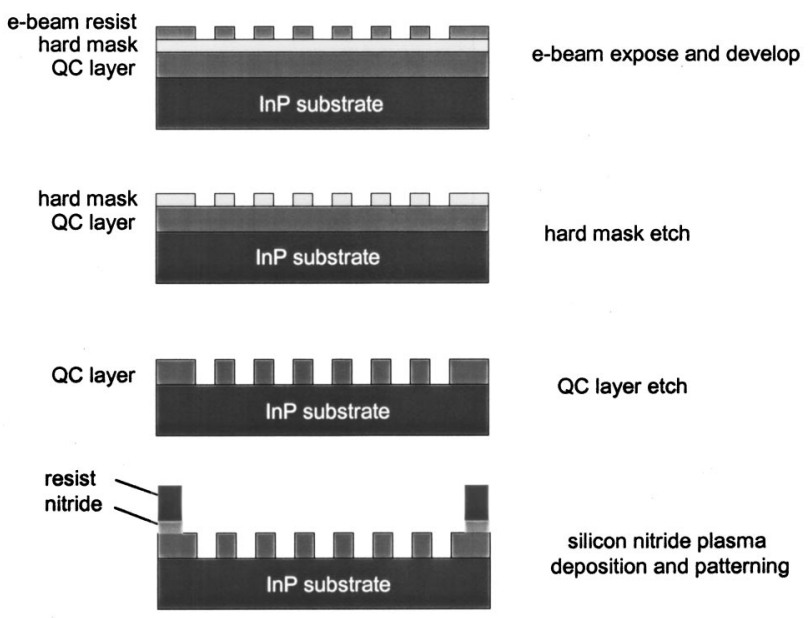

silicon nitride plasma deposition and patterning

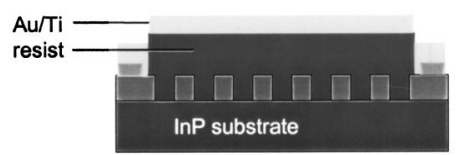

thick metal contact using positive tone photolithography

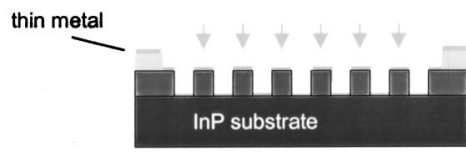

deposition of surface plasmon metal cladding/contact layer and device isolation

FIG. 1. Schematic of the major process steps used to produce photonic crystal Quantum Cascade devices.

which was developed as a $248 \mathrm{~nm}$ DUV resist, as shown in Fig. 2. The chemically amplified resist both exhibited more than adequate resolution and etch resistance, and in addition required significantly lower dose than conventional single component resists. This allowed us to better populate our samples with practical writing times. Two choices of hard mask material were compared, silicon nitride and silicon dioxide. Figure 3 shows that a much improved sidewall angle was obtained with silicon oxide. The patterned e-beam resist is transferred to the underlying silicon oxide hard mask layer via reactive ion etching using $\mathrm{CHF}_{3}$, typically 20-40 min

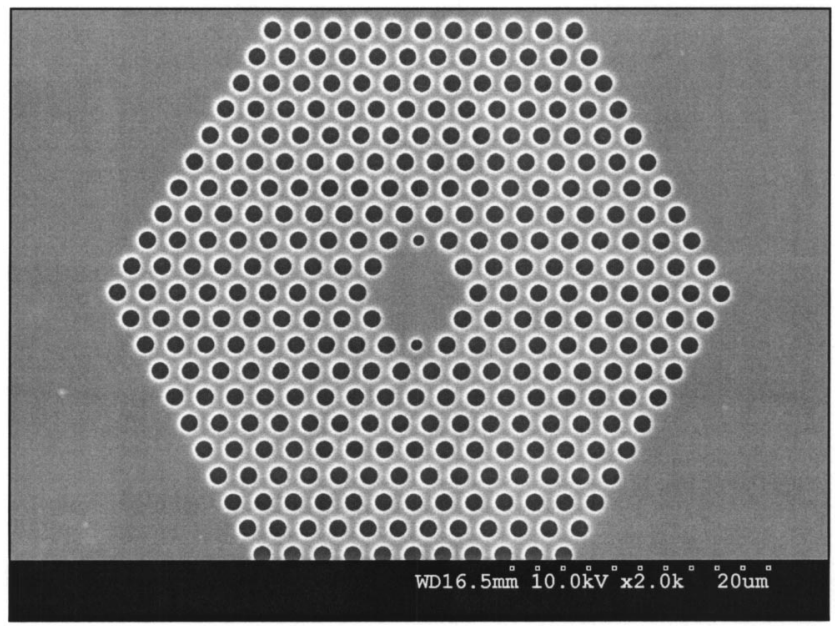

FIG. 2. Scanning electron micrograph of a typical photonic crystal pattern in UV 113 after ebeam exposure and development. 

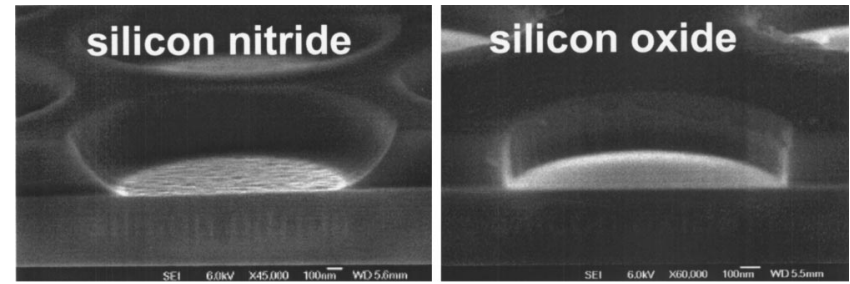

FIG. 3. Electron micrographs of silicon nitride (left) and silicon oxide (right) hard masks after etching. The improved sidewall slope exhibited by the oxide hard mask was helpful for the pattern transfer into the semiconductor. The image is of a cleave through a single hole in the photonic crystal pattern.

etch. The remaining resist is solvent removed. It proved critical to clean the polymeric buildup which occurs during the hard mask etching step to successfully transfer the PC pattern into the active QC layers. This is accomplished via a combination of oxygen plasma ashing $(\sim 50 \mathrm{~W}$ at $\approx 200$ mTorr for $10-15 \mathrm{~min}$ ) followed by a vigorous spray of acetone. To illustrate this, Fig. 4 shows the patterned hard mask before and after removal of the build up.

Once cleaned, the transfer to the semiconductor stack is achieved using a chlorine based inductively-coupled plasma reactive ion etch (ICP/RIE) which produces nearly $90^{\circ}$ wall as shown in Fig. 5. Typically heating to temperatures in the range $150-200{ }^{\circ} \mathrm{C}$ is needed to etch InGaAs/AlInAs and $\mathrm{InP}$. Although this etch is performed nominally at room temperature, the sample is sufficiently heated in situ by the plasma. Typical conditions are: $3 \mathrm{mT}$ pressure, mixture $\mathrm{Cl}_{2}: \mathrm{Ar}:: 8: 12 \mathrm{sccm}$, ICP power $300 \mathrm{~W}$ and rf power $200 \mathrm{~W}$. The steep angles are essential to allow use of a simple topside contact step described below.

Next, the remaining hard mask is removed using a second $\mathrm{CHF}_{3}$ etch followed by the cleaning step described above. The contact layer described below, is insulated from the substrate using a layer of plasma assisted silicon nitride. The nitride pattern therefore defines the region of current injection. The windows in the nitride are opened at the PC device sites using contact optical lithography with an image reversal process using AZ5214 (from Clariant). Image reversal was found to improve yield, as the severe topography creates difficulties for conventional positive resist processes. After that, a thick, low resistance Ti/Au (30/300 nm) contact was

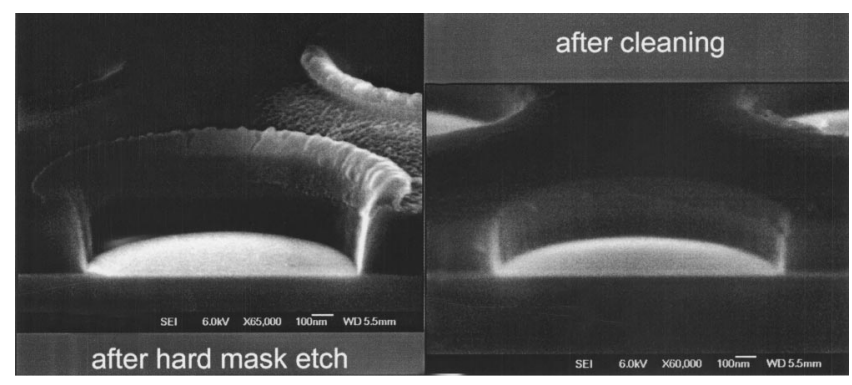

FIG. 4. Electron micrograph of the silicon hard mask before and after the cleaning step to remove polymeric buildup. The image is of a single hole in the photonic crystal pattern.

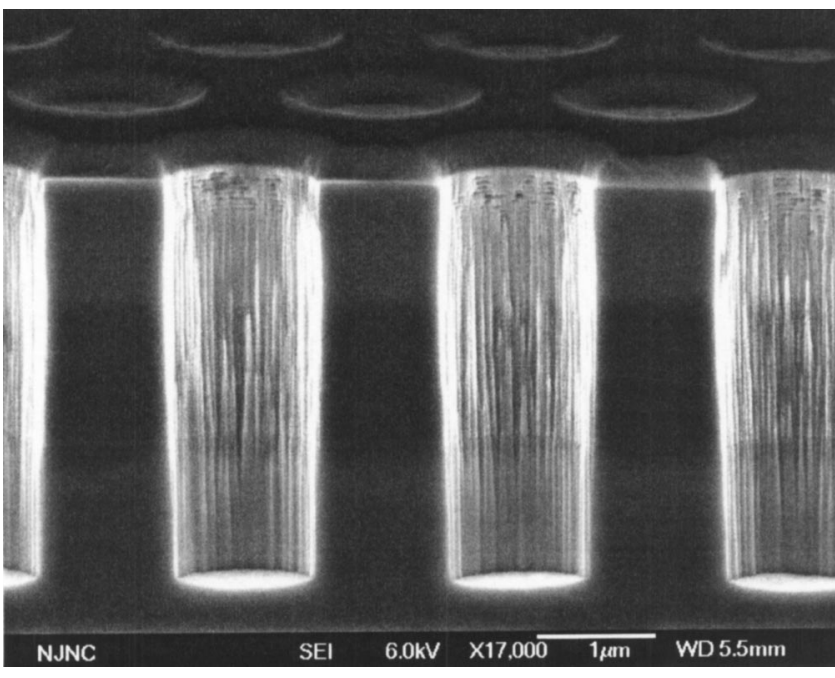

FIG. 5. Scanning electron micrograph of a cleaved device after the deep etch into the semiconductor showing that nearly $90^{\circ}$ sidewall angles were achieved.

patterned up to the border of the hexagonal device areas using liftoff from a single layer positive resist, (Shipley 1813) followed by the cleaning procedure. The sample is then thinned by lapping with an alumina polish from the back side followed by deposition of a metal back-contact comprised of e-beam evaporated $\mathrm{Au} / \mathrm{Ge} / \mathrm{Ag} / \mathrm{Au}$ in corresponding thicknesses of $12 / 27 / 50 / 300 \mathrm{~nm}$. Next a vertical evaporation and liftoff of thin Ti/Au $(10 / 100 \mathrm{~nm})$ is performed again using the image reversal described above. The thin Ti/Au contact plays a key role in this device. Normally pure gold is used as surface-plasmon carrying layer. We introduced a very thin layer of Ti for sticking purposes, after testing the procedure on a surface-plasmon QC laser processed as a regular stripe. In addition this thin cladding method aids in achieving a better current uniformity into the active area of the device. The stringent requirement for vertical holes in the active semiconductor etch is now evident. This deposition must provide the topside contact while preventing electrical shorting of the top contact to the substrate. Metal is thus deposited between the holes and on the bottom of the holes. It should not be deposited on the walls. Figure 6 shows a cross section of the holes after the topside deposition. Figure 7(b) shows the region where the thick bondable contact transitions to the thin top side contact after the complete process sequence.

\section{MEASUREMENTS AND DISCUSSION}

The photonic crystal pattern used for this study is a hexagonal region approximately $50 \mu \mathrm{m}$ across which contains hexagonally ordered dots of radius, $\mathbf{r}$, with nearest neighbor lattice constants, a. Figure 7(a) includes a representative completed device fabricated by the process described above. The effective refractive index in the QC material is not known with absolute precision since it is dependent on the detail conditions and thickness parameters. We therefore fabricated an array of devices in which the (photonic crystal) 


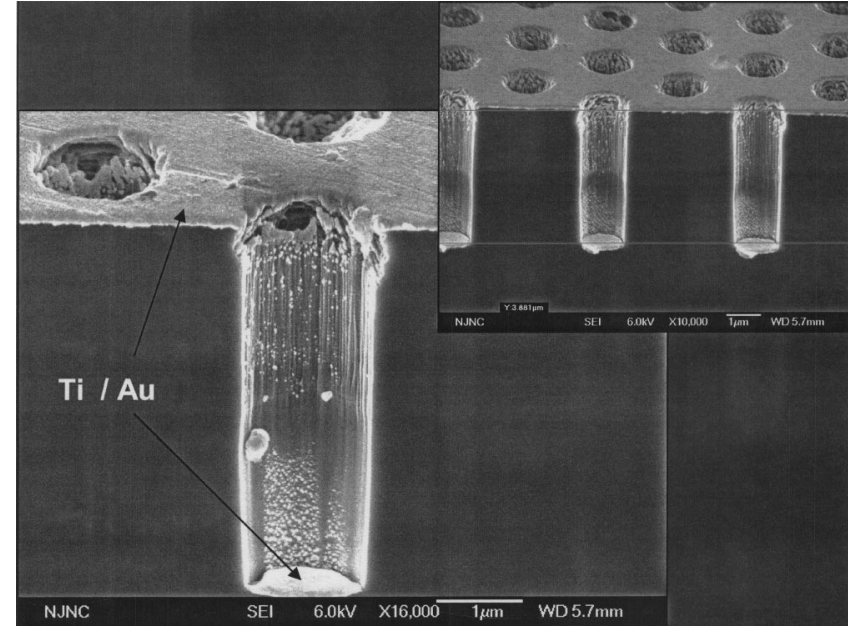

FIG. 6. Scanning electron micrograph of a cleaved device after the deposition of the top (thin) Au/Ti cladding. The steep sidewall prevents electrical shorting between the topside contact and the bottom of the holes.

lattice spacing and hole radius were systematically varied. This array is represented schematically in Fig. 8, which indicates the range over which the radii and lattice constants were varied. Down the columns, the values of $a$ were incremented and across the rows the $r / a$ ratio was incremented. This approach allowed us to tune the photonic crystal modes on top of the QC material gain spectrum peak by just choosing among the various devices in the array.

We fabricated two different types of PC QC devices, with and without a defect, to potentially operate them as both band-edge laser and defect mode (gap state) laser. The devices were measured at cryogenic temperatures $(10 \mathrm{~K})$ and light was collected vertically from the top surface of the device array. As seen in Fig. 9(a), when the PC is not in resonance with the QC material peak gain, the measured electroluminescence (EL) shows a broad peak centered at the intersubband emission wavelength of the QC heterostructure. The FWHM of the emission is equal to the one measured in a conventional edge-emitting stripe configuration at low currents: no gain-induced line narrowing and no sharp features

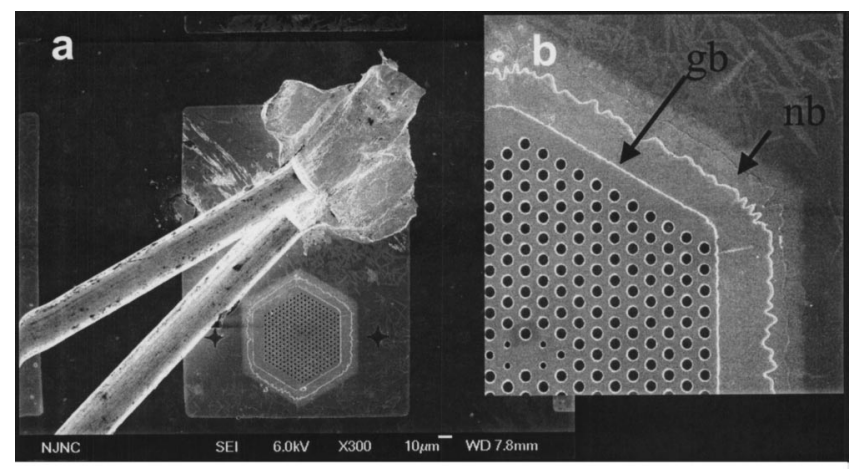

FIG. 7. (a) Completed device and (b) enlargement showing the photonic crystal area surrounded by a patterned nitride isolation and thick contact metal. The thick gold boundary, gb, and the nitride boundary, nb, are labeled.

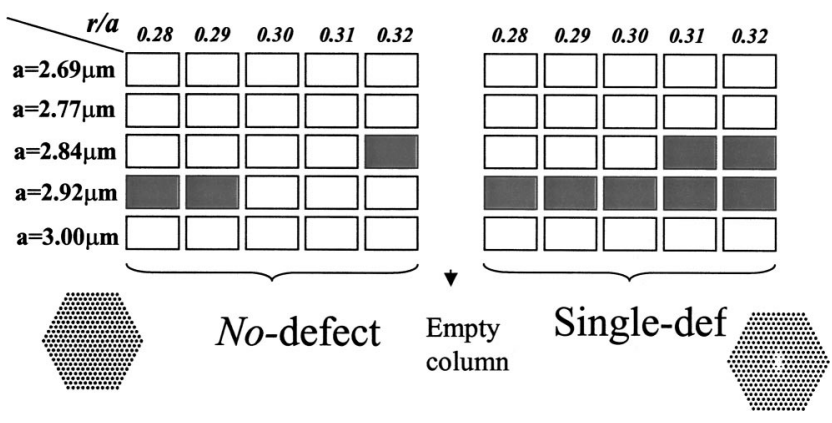

FIG. 8. Array of PC-QC laser structures were made in which the hole radius and lattice spacing were systematically varied to achieve the best resonance. Resonance was observed for the highlighted devices with the corresponding values of $r$ and $r / a$.

superimposed onto it. This spectrum is typical of the EL observed in the nonhighlighted sites in the device array in Fig. 8.

When there is a PC band-edge state in resonance with the QC material peak gain, a sharp peak appears on top of the broad EL emission [Fig. 9(b)]. As further proof that this feature is related to the photonic crystal band edge state, we can see it tuning with the radius and the lattice spacing [Figs. 9(c) and 9(d)]. Similarly resonant spectra were measured from the highlighted sites in Fig. 8.

\section{CONCLUSIONS}

We demonstrated a successful processing strategy to fabricate vertical emitting electrically pumped QC heterostructures based on PC technology. One of the key points was the use of a surface-plasmon waveguide for the QC devices. This technique allows us to use a shallower semiconductor etch $(4.7 \mu \mathrm{m}$ instead of $8 \mu \mathrm{m})$ since the active region of the QC device is closer to the top surface and the absence of the AlInAs claddings simplifies the ICP etch process. The pre-

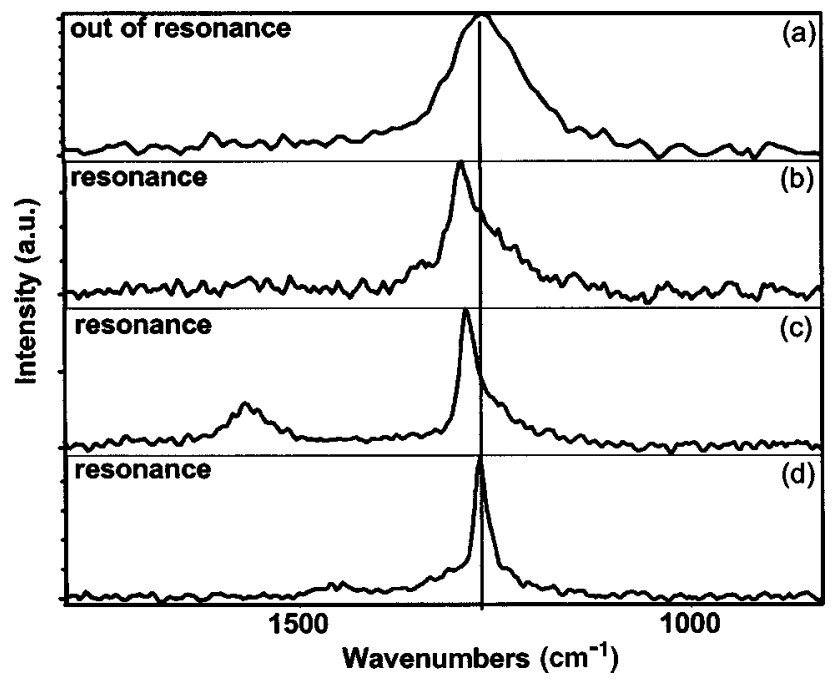

FIG. 9. Measured electroluminescence for off resonance (panel a), and for three different microresonators on resonance (panels b, c, and d). The shift between the spectrum in panel (b) and the one in panel (d) is $\approx 25 \mathrm{~cm}^{-1}$. 
cise control afforded by ebeam lithography to create the PC pattern allows fine adjustments to be implemented matching the resonance condition in the QC material.

The successful demonstration of these devices suggests that a new generation of mid-IR applications can be made available which take advantage of the possible miniaturization and implementation into two-dimensional arrays of QC lasers.

${ }^{1}$ J. Faist, F. Capasso, D. L. Sivco, C. Sirtori, A. L. Hutchinson, and A. Y. Cho, Science 264, 553 (1994).

${ }^{2}$ F. Capasso et al., IEEE J. Quantum Electron. 38, 511 (2002), and references therein.

${ }^{3}$ J. Faist, F. Capasso, D. L. Sivco, A. L. Hutchinson, S.-N. G. Chu, and A. Y. Cho, Appl. Phys. Lett. 72, 680 (1998); R. Colombelli et al., ibid. 78, 2620 (2001).

${ }^{4}$ R. Koehler et al., Nature (London) 417, 156 (2002).

${ }^{5}$ D. Hofstetter, J. Faist, M. Beck, and U. Oesterle, Appl. Phys. Lett. 75, 3769 (1999); W. Schrenk, N. Finger, S. Gianordoli, L. Hvozdara, G. Strasser, and E. Gornik, ibid. 77, 2086 (2000).

${ }^{6}$ J. D. Joannopoulos, P. R. Villeneuve, and S. H. Fan, Nature (London) 386, 143 (1997).
${ }^{7}$ J. D. Joannopoulos, R. D. Meade, and J. N. Winn, Photonic Crystals (Princeton University Press, Princeton, 1995).

${ }^{8}$ A. Scherer, J. L. Jewell, and J. P. Harbison, Opt. Photonics News 2, 9 (1991).

${ }^{9}$ W. W. Bewley, C. L. Felix, I. Vurgaftman, R. E. Bartolo, J. R. Lindle, J. R. Meyer, H. Lee, and R. U. Martinelli, Solid-State Electron. 46, 1557 (2002).

${ }^{10}$ I. Vurgaftman and J. R. Meyer, IEEE J. Quantum Electron. 38, 592 (2002).

${ }^{11}$ O. Painter, R. K. Lee, A. Scherer, A. Yariv, J. D. O’Brien, P. D. Dapkus, and I. Kim, Science 284, 1819 (1999).

${ }^{12}$ M. Imada, S. Noda, A. Chutinan, T. Tokuda, M. Murata, and G. Sasaki, Appl. Phys. Lett. 75, 316 (1999).

${ }^{13}$ H. G. Park, J.-K. Hwang, J. Huh, H.-Y. Ryu, Y.-H. Lee, and J.-S. Kim, Appl. Phys. Lett. 79, 3032 (2001).

${ }^{14}$ M. Notomi, H. Suzuki, and T. Tamamura, Appl. Phys. Lett. 78, 1325 (2001).

${ }^{15}$ I. Vurgaftman and J. R. Meyer, IEEE J. Quantum Electron. 39, 689 (2003).

${ }^{16} \mathrm{R}$. Colombelli et al., "Quantum cascade surface-emitting photoniccrystal laser," Science Express (published \& on-line) 30 October 2003.

${ }^{17}$ C. Gmachl, A. Tredicucci, F. Capassso, A. L. Hutchinson, D. L. Sivco, J. N. Baillargeon, and A. Y. Cho, Appl. Phys. Lett. 75, 316 (1999).

${ }^{18}$ C. Sitori, C. Gmachl, F. Capasso, J. Faist, D. L. Sivco, A. L. Hutchinson, and A. Y. Cho, Opt. Lett. 23, 1366 (1998). 